\title{
COMPUTATION OF AXISYMMETRIC VIBRATION TRANSMISSION USING A WELL-CONDITIONED SYSTEM FOR ELASTIC LAYERS OVER A HALF-SPACE
}

\author{
Andrew T. Peplow ${ }^{1}$, Lars V. Andersen ${ }^{2}$, and Peter Persson ${ }^{3}$ \\ ${ }^{1}$ Department of Natural Sciences and Public Health, Zayed University \\ P.O. Box 144534, Abu Dhabi, United Arab Emirates \\ e-mail: andrew.peplow@zu.ac.ae \\ ${ }^{2}$ Department of Engineering, Aarhus University \\ 8000 Aarhus C, Denmark \\ e-mail: lva@eng.au.dk \\ 3 3Department of Construction Sciences, Lund University \\ P.O. Box 118, SE-221 00 Lund, Sweden \\ e-mail: peter.persson@construction.lth.se
}

Keywords: Elastic wave propagation, axisymmetric, dynamic stiffness matrix.

\begin{abstract}
In the context of range-independent solid media, we propose a well-conditioned dynamic stiffness matrix for an elastic layer sitting over an elastic half-space. This formulation overcomes the well-known problem of numerical ill-conditioning when solving the system of equations for deep-layered strata. The methodology involves the exact solutions of transformed ordinary differential equations in the wavenumber domain, namely a projection method based on the transformed equations with respect to the depth coordinate. By re-arranging the transformed equations, the solutions remain numerically well-conditioned for all layer depths. The inverse transforms are achieved with a numerical quadrature method and the results presented include actual displacement fields in the near-field of the load in plane-strain and threedimensional axisymmetric cases. Verification against finite element method (FEM) calculations demonstrates the performance and complexity of the two approaches.
\end{abstract}




\section{INTRODUCTION}

A three-dimensional axisymmetric model is considered which demonstrates the effect of a harmonic finite rigid disk-load over various layered strata. The results derived by Fourier transformation are valid for any frequency and, more importantly, for any depth of the layer. In principle, following the well-known traditional methods, [1], we could use displacement-continuity and traction-equilibrium boundary conditions at the bottom and top of a layer with equations at the ground surface to generate equations for six subsequent unknowns of stress and displacement. However, this direct approach leads to formidable numerical problems. The reason for this work is that if traditional expressions for the characteristic wave functions, such as cosh or sinh, are employed, these can have a dramatic effect on the numerical evaluation of solutions. Problems arise due to the cancellation or division of either very small or very large numbers. To overcome this, Karasalo [2] derived a well-conditioned propagator matrix for radially symmetric problems. Recent advances in propagator-matrix techniques have proved successful, [3] and [4]; but we derive a simple and robust technique. In this work, then, we construct a single stiffness matrix for the physical layer for plane-strain problems which conveniently avoids these difficulties. We therefore deduce a new global dynamic stiffness matrix for functions that do not cause numerical problems on a desktop PC.

Although not developed in this work, solutions to characteristic equations, such as dispersion equations, which establish wave propagation parameters, can now be determined more efficiently. Similar work in this area for dynamic problems where the load is stationary has been presented in [6] or for general moving-load problems in [7]. In this work we show how we are able to solve large (read: deep) problems, due to the reduced number of equations.

\section{VIBRATION TRANSMISSION}

The model considered is shown in Figure 1(a). A rigid disk load has a radius $b>0$, with respect to the $r$-axis. It rests on homogeneous, isotropic, axisymmetric elastic layers, with material properties $E$ (Young's modulus), $\rho$ (density) and $\nu$ (Poisson's ratio). A harmonic vertical load acts uniformly over the disk. A submerged elastic layer (the concrete block) of finite depth, $H>0$, of homogeneous and isotropic material then overlies a half-space of flexible material. The block is considered as a finite-width disk, radius $L>0$, or as having infinite width, which is a similar problem to one studied in [8].

\subsection{Geometry}

The problem is solved semi-analytically by a numerical quadrature method suited for oscillatory integrands and, for verification and parametric analysis, by COMSOL 5.2 Solid Structures analysis. For the benefit of the reader, Fig. 1(b) includes an illustration of a typical mesh for the problem in the axisymmetric case. The model has 37, 454 degrees of freedom (d.o.f.), 4, 064 domain elements, and 611 boundary elements with cubic shape functions. In this example, the perfectly matched layer (PML) region surrounds the solution zone at $R=75.0 \mathrm{~m}$, and receiver positions of interest are at $R_{1}=4.0 \mathrm{~m}, R_{2}=16.0 \mathrm{~m}$, and $R_{3}=32.0 \mathrm{~m}$.

\subsection{Theoretical background}

Much of the analysis necessary for the derivation of the dynamic stiffness matrix has been presented in references [9] and [10], so this will only be briefly summarized. For plane strain, the behaviour of the isotropic elastic material is described by Navier's elastodynamic equations, 


\section{(a)}

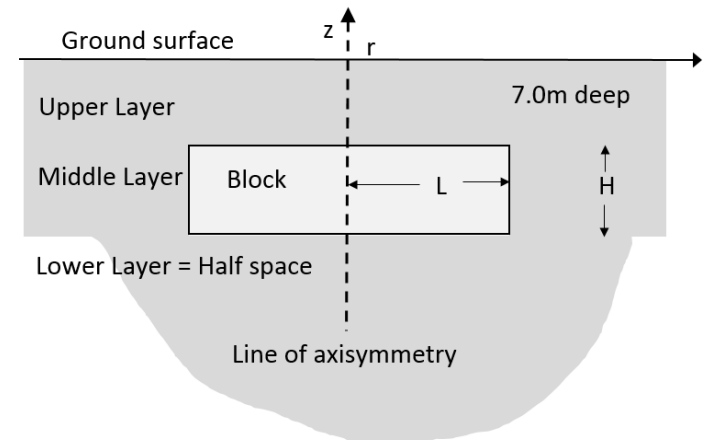

(b)

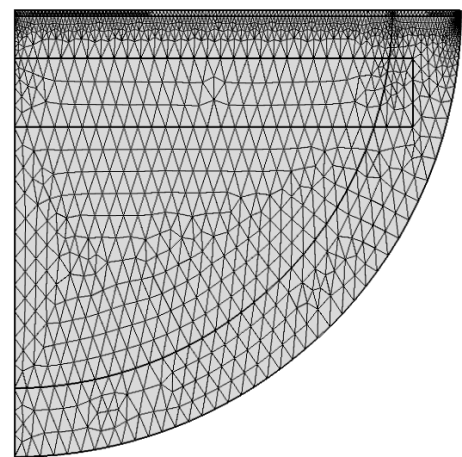

Figure 1: The schema for the two approaches: (a) For the semi-analytical domain, block has radius $L \rightarrow \infty$; (b) Axisymmetry model from COMSOL 5.2 showing the block of finite radius $L=70 \mathrm{~m}$ penetrating the PML region within the finite element mesh.

[9]. In the absence of body forces, these can be written as:

$$
\begin{aligned}
(\lambda+\mu) \frac{\partial e}{\partial z^{2}}+\mu\left(\frac{\partial^{2} u}{\partial r^{2}}+\frac{1}{r} \frac{\partial u}{\partial r}-\frac{u}{r^{2}}+\frac{\partial^{2} u}{\partial z^{2}}\right) & =\rho \frac{\partial^{2} u}{\partial t^{2}} \\
(\lambda+\mu) \frac{\partial e}{\partial z}+\mu\left(\frac{\partial^{2} w}{\partial r^{2}}+\frac{1}{r} \frac{\partial w}{\partial r}+\frac{\partial^{2} w}{\partial z^{2}}\right) & =\rho \frac{\partial^{2} w}{\partial t^{2}}
\end{aligned}
$$

where $u, w$ are the components of the displacement in the $r$ and $z$ directions and $e$ is the volume strain, and $\lambda, \mu$ are complex Lamé constants assuming a constant loss-factor damping model. The quantities $c_{1}$ and $c_{2}$ are, respectively, the $P$ and $S$ wave speeds, given by:

$$
c_{1}^{2}=\frac{\lambda+2 \mu}{\rho}=\frac{E(1-\nu)}{\rho(1+\nu)(1-2 \nu)}, \quad c_{2}^{2}=\frac{\mu}{\rho}=\frac{E}{2 \rho(1+\nu)} .
$$

and the boundary conditions for this problem are a uniform rigid disk-load of width $b$ acting over the surface and at, for the example given here, at $z=H_{1}, z=\left(H_{1}+H_{2}\right)$ continuity of displacement and equilibrium of traction between the mid-layer and the upper and lower media. Equations (2) may be solved by introducing potentials $\phi$ and $H$ such that

$$
u=\frac{1}{r} \frac{\partial \phi}{\partial r}-\frac{\partial H}{\partial z} \quad \text { and } \quad w=\frac{\partial \phi}{\partial z}+\frac{1}{r} \frac{\partial H}{\partial r} .
$$

Also, assuming harmonic time-dependence $\mathrm{e}^{\mathrm{i} \omega t}$ and axial symmetry of the problem, this suggests seeking solutions in the form of Hankel integrals. For this purpose, the following Hankel transforms are introduced, assuming $\zeta$ is the transform variable:

$$
\bar{U}=\int_{0}^{\infty} u r J_{1}(\zeta r) d r \text { and } \bar{W}=\int_{0}^{\infty} w r J_{0}(\zeta r) d r
$$

Taking the Hankel transform of the frequency version of equations (1) and (2) leads to a coupled set of ordinary differential equations.

$$
\begin{aligned}
& \mu \frac{d^{2} \bar{U}}{\partial z^{2}}+\left[(\lambda+2 \mu) \zeta^{2}-\rho \omega^{2}\right] \bar{U}-(\lambda+\mu) \zeta \frac{d \bar{W}}{\partial z}=0 \\
& (\lambda+2 \mu) \frac{d^{2} \bar{W}}{\partial z^{2}}-\left[\mu \zeta^{2}-\rho \omega^{2}\right] \bar{W}-(\lambda+\mu) \zeta \frac{d \bar{U}}{\partial z}=\operatorname{Pr} J_{0}(\zeta r) .
\end{aligned}
$$




\begin{tabular}{lcc}
\hline Soil & $\begin{array}{c}\text { Upper-layer and half-space } \\
\text { Soft soil-type }\end{array}$ & $\begin{array}{c}\text { Embedded block } \\
\text { Concrete }\end{array}$ \\
\hline$E(\mathrm{~Pa})$ & $2.69 \times 10^{8}$ & $204 \times 10^{8}$ \\
$\rho\left(\mathrm{kg} \mathrm{m}^{-3}\right)$ & 1550 & 2450 \\
$\nu$ & 0.257 & 0.179 \\
$c_{R}\left(\mathrm{~m} \mathrm{~s}^{-1}\right)$ & 242 & 1706 \\
$c_{2}\left(\mathrm{~m} \mathrm{~s}^{-1}\right)$ & 263 & 1879 \\
$c_{1}\left(\mathrm{~m} \mathrm{~s}^{-1}\right)$ & 459 & 3005 \\
\hline
\end{tabular}

Table 1: Material properties

It is common practice to write solutions to the ensuing ordinary differential equations over a finite-length domain, $z \in[0, D]$ in terms of cosh and sinh functions. For computational purposes, this choice of characteristic functions is not convenient for problems involving spatial domains chosen to be extremely deep. Hence, we propose the general solutions may be written as a scaled formulation:

$$
\begin{gathered}
\bar{\phi}=A_{1} \mathrm{e}^{-\alpha_{1} z}+A_{2} \mathrm{e}^{\alpha_{1}(z-D)}, 0 \leq z \leq D \\
\bar{H}=B_{1} \mathrm{e}^{-\alpha_{2} z}+B_{2} \mathrm{e}^{\alpha_{2}(z-D)}, 0 \leq z \leq D . \\
\alpha_{1}^{2}=\zeta^{2}-k_{1}^{2} \quad \text { and } \quad \alpha_{2}^{2}=\zeta^{2}-k_{2}^{2} .
\end{gathered}
$$

The reasons for choosing the scaled exponential characteristic functions over the hyperbolic functions is clear. Essentially, this choice ensures the characteristic functions do not grow unbounded with depth. Substituting the values $z=0$ and $z=D$ into the equations (8) yields the first matrix equation

$$
\{\bar{u}\}=[C]\{A\} .
$$

where $\bar{u}=\left[\mathrm{i} \bar{w}_{0}, \bar{u}_{0}, \mathrm{i} \bar{w}_{D}, \bar{u}_{D}\right]^{T}$ and the $4 \times 4$ complex-valued matrix $[C]$ is given by

$$
[C]=\left[\begin{array}{llll}
-\mathrm{i} \alpha_{1} & \mathrm{i} \alpha_{1} \mathrm{e}^{-\alpha_{1} D} & -\zeta & -\zeta \mathrm{e}^{-\alpha_{2} D} \\
\mathrm{i} \zeta & \mathrm{i} \zeta \mathrm{e}^{-\alpha_{1} H} & \alpha_{2} & -\alpha_{2} \mathrm{e}^{-\alpha_{2} D} \\
-\mathrm{i} \alpha_{1} \mathrm{e}^{-\alpha_{1} D} & \mathrm{i} \alpha_{1} & -\zeta \mathrm{e}^{-\alpha_{2} D} & -\zeta \\
\mathrm{i} \zeta \mathrm{e}^{-\alpha_{1} D} & \mathrm{i} \zeta & \alpha_{2} \mathrm{e}^{-\alpha_{2} D} & -\alpha_{2}
\end{array}\right]
$$

Now, further developing the system of equations, we get

$$
\begin{gathered}
\{\bar{\sigma}\}=[S]\{A\} \\
{[S]=\left[\begin{array}{llll}
-\mathrm{i} \alpha_{1}^{2} \beta+\mathrm{i} \lambda \zeta^{2} & \left(-\mathrm{i} \alpha_{1}^{2} \beta+\mathrm{i} \lambda \zeta^{2}\right) g_{1} & -2 \mu \zeta \alpha_{2} & 2 \mu \zeta \alpha_{2} \zeta g_{2} \\
2 \mathrm{i} \mu \zeta \alpha_{1} & -2 \mathrm{i} \mu \zeta \alpha_{1} \zeta g_{1} & \mu\left(\alpha_{2}^{2}+\zeta^{2}\right) & \mu\left(\alpha_{2}^{2}+\zeta^{2}\right) g_{2} \\
\mathrm{i}\left(\alpha_{1}^{2} \beta-\lambda \zeta^{2}\right) g_{1} & \mathrm{i}\left(\alpha_{1}^{2} \beta-\lambda \zeta^{2}\right) & 2 \mu \zeta \alpha_{2} g_{2} & -2 \mu \zeta \alpha_{2} \\
-2 \mathrm{i} \mu \zeta \alpha_{1} g_{1} & 2 \mathrm{i} \mu \zeta \alpha_{1} & -\mu\left(\alpha_{2}^{2}+\zeta^{2}\right) g_{2} & -\mu\left(\alpha_{2}^{2}+\zeta^{2}\right)
\end{array}\right]}
\end{gathered}
$$

with $\beta=\lambda+2 \mu, g_{i}=\mathrm{e}^{-\alpha_{i} D}, i=1,2$, and $\bar{\sigma}=\left[-\mathrm{i} \bar{\sigma}_{0},-\bar{\tau}_{0}, \mathrm{i} \bar{\sigma}_{D}, \bar{\tau}_{D}\right]^{T}$. We now combine equations (9) and (11), to arrive at a single matrix expression which expresses the displacements and stresses at the surface and the interface in the wavenumber domain:

$$
[T]\{\bar{u}\}=\{\bar{\sigma}\}
$$


where $[T]=[S][C]^{-1}$. The entries of the matrix $[T]$ are provided in the work of Peplow, [5]. Specifically, $[T]$ is the dynamic stiffness matrix for a single elastic layer, valid for any layerdepth $D>0$. To include the half-space, we utilize the matrix equations presented in [10], which leads to a matrix system:

$$
[P]=\frac{1}{D E T}\left[\begin{array}{cc}
(\lambda+2 \mu) \alpha_{2} k_{1}^{2} & 2 \mu \zeta\left(\alpha_{1} \alpha_{2}-\zeta^{2}\right)+(\lambda+2 \mu) \zeta k_{1}^{2} \\
2 \mu \zeta\left(\alpha_{1} \alpha_{2}-\zeta^{2}\right)+\mu \zeta k_{2}^{2} & \mu \alpha_{1} k_{2}^{2}
\end{array}\right]\left[\begin{array}{l}
\mathrm{i} \bar{w}_{D} \\
\bar{u}_{D}
\end{array}\right]=\left[\begin{array}{l}
\mathrm{i} \bar{\sigma}_{D} \\
\bar{\tau}_{D}
\end{array}\right]
$$

where $D E T=1 /\left(\alpha_{1} \alpha_{2}-\zeta^{2}\right)$ and it is understood the soil parameters are related to the halfspace below the upper layer.

Equations can now be combined to give a single matrix equation for an elastic layer over an elastic half-space, involving the scaled stiffness matrix for the elastic layer $\left[T_{i j}\right]$ and the matrix for the half-space $\left[P_{i j}\right]$. The general matrix form for any global domain becomes a $4 \times 4$ complex valued matrix:

$$
[T]_{G}=\left[\begin{array}{cc:cc}
T & T & T & T \\
T & T & T & T \\
\hdashline T & T & T-P & T-P \\
T & T & T-P & T-P
\end{array}\right]
$$

It is straightforward then to generalise this technique to $n$ elastic layers over a half-space, where the size of the dynamic stiffness matrix will become a single complex-valued $2(n+1) \times 2(n+1)$ matrix. On our case we have essentially two layers on a half-space $(n=2)$, which results in a $6 \times 6$ system.

\section{NUMERICAL COMPUTATIONS}

Generally, for non-dimensional wavenumbers $k_{s} h \geq 13$, where $k_{s}$ is the shear wavenumber, the conventional approach "breaks down". That is, for depths greater than around two shear wavelengths, $h \geq 2 \lambda_{s}$, a numerical bottleneck problem arises when solving the linear system of algebraic equations. Equally, for high frequencies, computations can become ill-conditioned. The choice of projected method permits a stable numerical evaluation for entries in the stiffness matrix for all soil types, frequencies and layer depths. This avoids numerical round-off errors.

It was found that numerical inversion of the Hankel transforms worked well with a cut-off at $R=20 \mathrm{~m}^{-1}$ with 2,000 sample points spaced logarithmically in the interval $\zeta \in\left[10^{-4}, R\right]$. Fast numerical Hankel methods, similar to Fast Fourier Transform (FFT), which are freely available, were not used in this work since our interest is in a few receiver positions.

\section{RESULTS}

Soil parameters used are presented in Table 1, that is two sets of soil characteristics which represent different conditions, soil and concrete. The response has been calculated for a diskload with $b=1.0 \mathrm{~m}$ and subjected to a uniform unit load, $P=1 \mathrm{~N}$. Calculation times for 100 frequency points on an i3-chip desktop took around 3.0 minutes in total. Integration was not particularly slow due to the modes associated to the deep bedrock stratum. Numerical integration is possible for all deep models and it is recommended to include an underlying halfspace for computational and realistic modelling purposes, since bedrock models will include many modes.

Nonetheless the results presented are calculated using the Clenshaw-Curtis numerical quadrature method. The related ground parameters are shown in Table 1. The configuration is an elastic 
circular block layer embedded $7.0 \mathrm{~m}$ deep within a half-space. To verify results of the simple model, we compare with a numerical scheme provided by COMSOL 5.2, see Fig. 1(b).

(a)
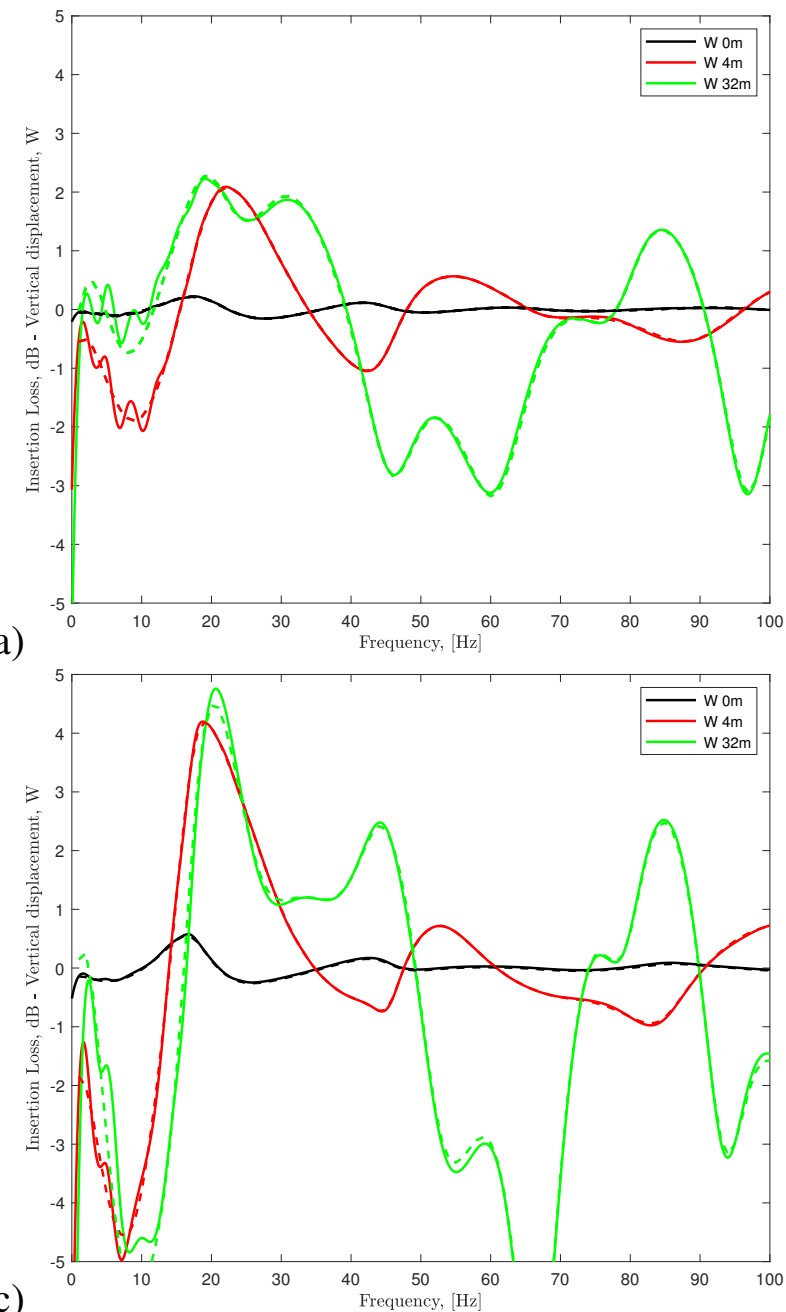

(b)

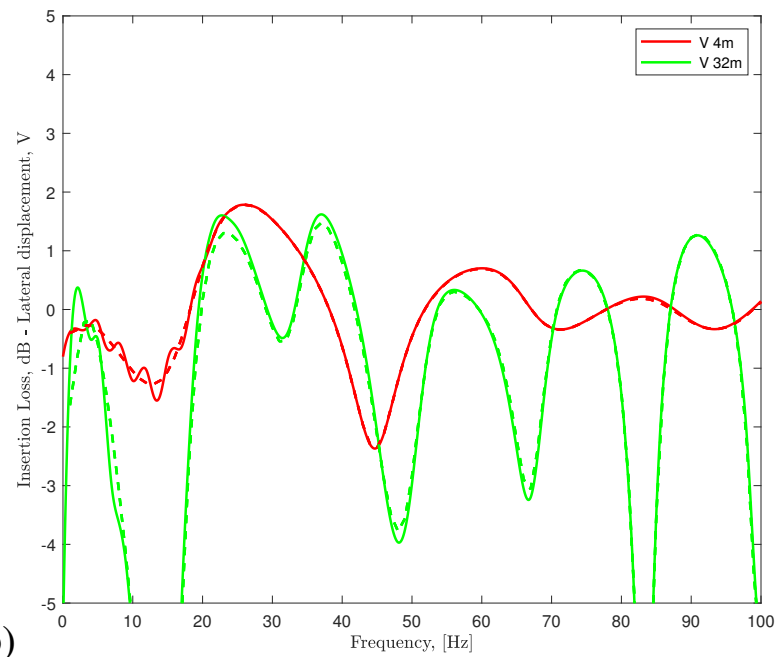

(d)

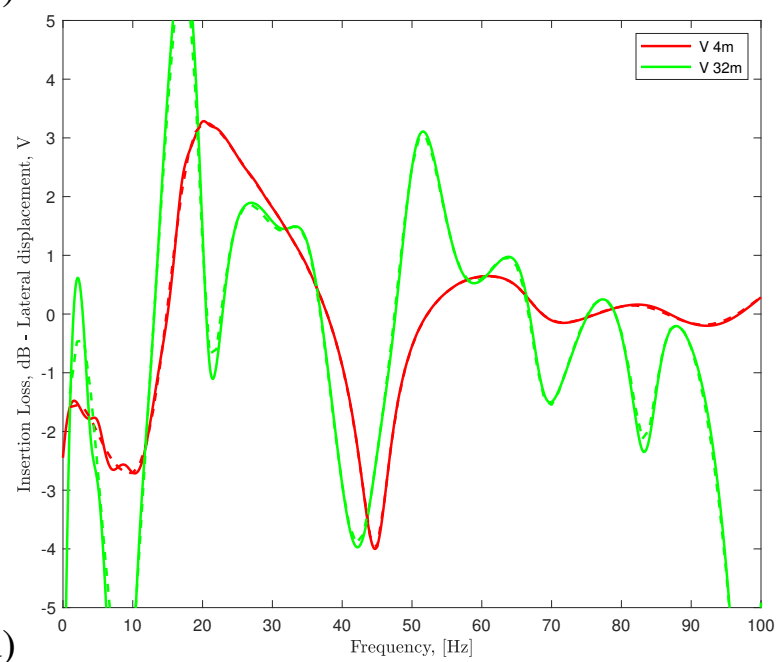

Figure 2: Verification of the method compared to a PML version in COMSOL 5.2 for: (a) and (b) $0.5 \mathrm{~m}$ deep embedded concrete block; and (b) and (c) a 2.0 m embedded block.

Our approach, as a possible measure for mitigating vibration, is based on the impeding behaviour of a soil layer over a bedrock. It occurs if the excitation frequency is below the lowest eigenfrequency of the layer. Since it is not convenient to develop real bedrock layers below buildings, where the length of the block in Fig. 1 tends to infinity, for example, we may use a stiff finite-length obstacle as an artificial bedrock instead. The results correspond to the possible positive effect for replacing bedrock by a feasible concrete block. For a bedrock layer at depth, $H=7.0 \mathrm{~m}$, the first eigenfrequency related to shear waves is around $10 \mathrm{~Hz}$. We only show two examples where the rigid bedrock is approximated by a concrete block, $0.5 \mathrm{~m}$ thick, with various lengths and evaluated at three receiver positions.

Figures 3(a) and (b) correspond to amplitude increase or decrease, against the half-space response, as the thin $0.5 \mathrm{~m}$ concrete block increases width from $L=2.0 \mathrm{~m}$, to $L=4.0 \mathrm{~m}$ to $L=12.0 \mathrm{~m}$ with respect to the receiver position $4.0 \mathrm{~m}$ from the load-centre. For both vertical and longitudinal motion we observe a characteristic dip in insertion loss below the cut-on frequency with a slight insertion loss up to $2 \mathrm{~dB}$ performance in the interval $20 \mathrm{~Hz}$ to 
$40 \mathrm{~Hz}$. A concrete block of length $12.0 \mathrm{~m}$ appears to approximate well as a substitute for an infinite layer. Figures 3 (c) and (d) correspond to amplitude increase or decrease as the thin 0.5 m concrete block increases width from $L=8.0 \mathrm{~m}, L=16.0 \mathrm{~m}$ to $L=24.0 \mathrm{~m}$ with respect to the receiver position $16.0 \mathrm{~m}$ from the load-centre. Here, the complex interactions below cuton are evident, again a possible $2 \mathrm{~dB}$ insertion loss between $20 \mathrm{~Hz}$ and $40 \mathrm{~Hz}$ seems possible. Figures 3 (e) and (f) correspond to amplitude increase or decrease as the thin $0.5 \mathrm{~m}$ concrete block increases width from $L=24.0 \mathrm{~m}, L=32.0 \mathrm{~m}$ to $L=40.0 \mathrm{~m}$ with respect to the receiver position $32.0 \mathrm{~m}$ from the load-centre. As the receiver position increases its distance from the load, the concrete block, substituting a rigid layer foundation, also requires greater length Again the possible effectiveness as a mitigation device reduces to below $40 \mathrm{~Hz}$.

\section{CONCLUSIONS}

An axisymmetric three-dimensional model has been developed for investigating the propagation of surface vibration over arbitrary-depth elastic layers. The model consists of an elastic, isotropic and homogeneous layer which overlies a half-space. A well-conditioned dynamic stiffness matrix has been developed for this model, which is derived by considering a different set of characteristic functions.

We have shown that by including a bedrock foundation buried under a surface load this can have a positive effect in reducing vibration across the surface of the ground, in certain frequency bands. However, below the cut-on frequency, in which travelling waves cannot propagate, the effectiveness is difficult to assess.

Rather than turning to large-scale finite element models we have shown that it is possible to draw conclusions for vibration reduction assessments from an alternative general semianalytical model. In future work we shall extend the semi-analytical model illustrated in this work from the axisymmetric case to general three-dimensional models.

\section{ACKNOWLEDGEMENTS}

The third author gratefully acknowledges the financial support from the Swedish Governmental Agency for Innovation Systems (Vinnova), grant ref.no. 2018-04159, as well as from the City of Helsingborg through the call Plattformen.

\section{REFERENCES}

[1] D.V. Jones, M. Petyt, Ground vibration in the vicinity of a strip load: an elastic layer on an elastic half-space. Journal of Sound and Vibration, 161, 1-18, 1993.

[2] I. Karasalo, Exact finite elements for wave propagation in range-independent fluid-solid media. Journal of Sound and Vibration, 172, 671-688, 1994.

[3] J.F. Lu, R.F. Shen, M.Q. Sh, Reflection-transmission matrix method for the consolidation of a multilayered saturated soil. International Journal for Numerical and Analytical Methods in Geomechanics, 40, 1594-1622, 2016.

[4] C.H. Chapman, Yet another elastic plane-wave, layer-matrix algorithm. Geophysical Journal International, 154, 212-223, 2003.

[5] A.T. Peplow, Surface ground vibration from a stripload acting over elastic layers: A uniform approach. Soil Dynamics and Earthquake Engineering, 116, 289-292, 2019. 
(a)

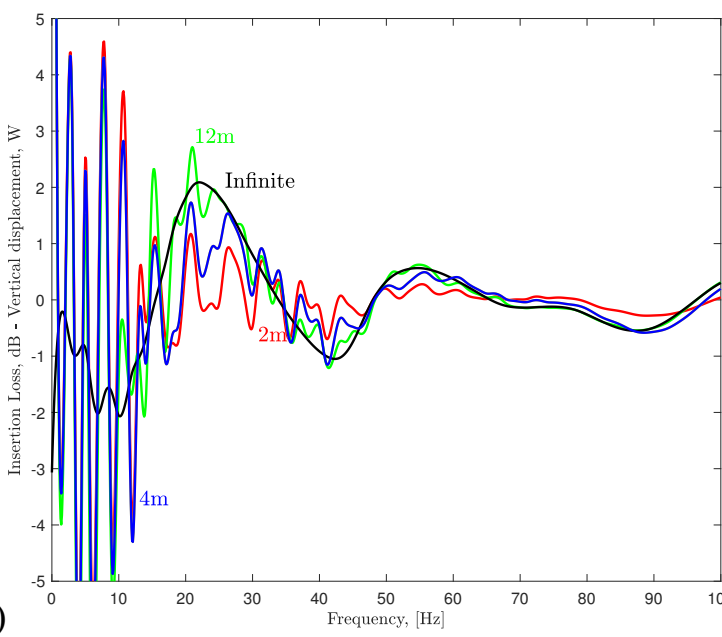

(c)

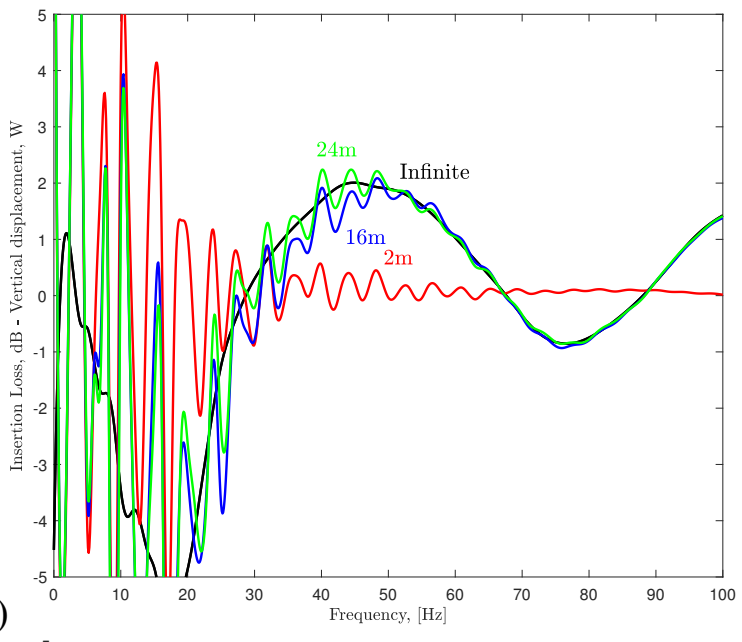

(d)

(b)
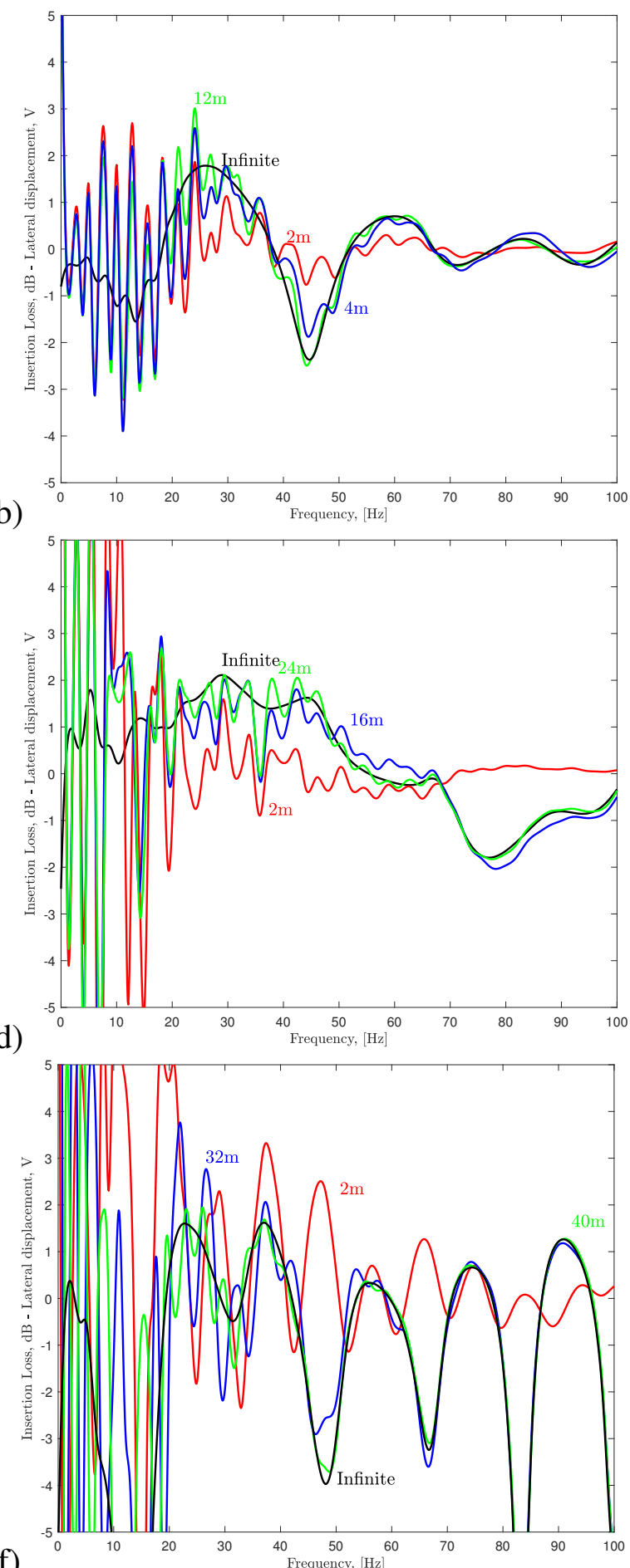

(e)

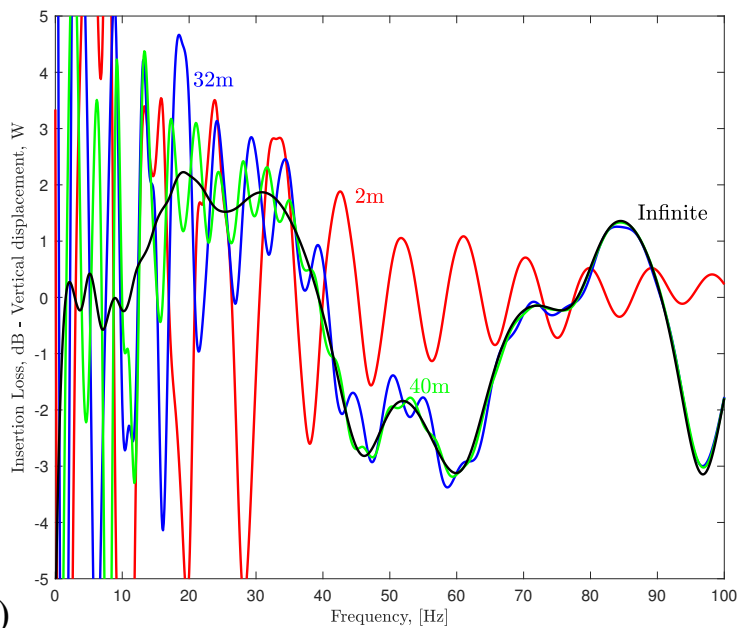

(f)

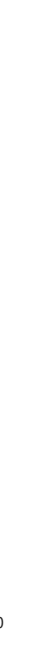

Figure 3: Convergence of insertion loss for various finite-radius embedded concrete blocks: (a), (b) at receiver $4.0 \mathrm{~m}$ from load-centre; (c), (d) at $16.0 \mathrm{~m}$ from load-centre; and (e), (f) at $32.0 \mathrm{~m}$ from load-centre. Also shown results from the simple model for an infinite radius disk.

[6] C.L. Liu, Z.Y. Ai, Vertical harmonic vibration of piled raft foundations in layered soils. International Journal for Numerical and Analytical Methods in Geomechanics, 41, 17111723, 2017. 
[7] H. Sun, Y. Cai, C. Xu, Three-dimensional steady-state response of a railway system on layered half-space soil medium subjected to a moving train. International Journal for Numerical and Analytical Methods in Geomechanics, 33, 529-550, 2009.

[8] A.T. Peplow, M. Petyt, C.J.C. Jones, Surface vibration propagation over a layered halfspace with an inclusion. Applied Acoustics, 56, 283-296, 1999.

[9] W.M. Ewing, W.S Jardetzky, F. Press, Elastic waves in layered media, McGraw-Hill, New York, 1957.

[10] D.V. Jones, M. Petyt, Ground vibration in the vicinity of a strip load: A two-dimensional half-space model. Journal of Sound and Vibration, 147, 155-166, 1991. 